\title{
Cours d'introduction
}

\author{
C. Fermon
}

DSM/DRECAM/SPEC, CEA/Saclay, 91191 Gif-sur-Yvette, France

\section{INTRODUCTION}

Ce cours d'introduction est destiné à poser les bases utiles pour la compréhension des journées d'enseignement sur le magnétisme et les neutrons. Ce cours commence à partir de la fonction d'onde du neutron.

Il peut vous apparaître par certains côtés très formel et difficilement accessible pour ceux qui ne sont pas habitués à la mécanique quantique. Pour cela les parties encadrées sont celles à retenir et qui seront reprises dans les cours suivants.

Cette première partie est destinée à ceux qui connaissent la mécanique quantique et qui veulent savoir comment ont été obtenus les facteurs de structures et les formules d'interaction magnétiques. Pour ceux qui ne sont pas familiers avec la mécanique quantique, ils peuvent se rattacher directement aux équations 33 et 34 .

Dans toute la première partie je ne traite que le cas élastique, lorsque l'énergie des neutrons n'est pas modifiée par la diffusion sur les atomes. Ce sera le cas en réflectivité, diffusion aux petits angles et diffraction. La fin de cette première partie aboutit au facteurs de structure.

La relation entre intensité mesurée et les fonctions de corrélations est traité en annexe 1. Cette annexe utilise le formalisme des distributions tel qu'il peut être enseigné à un niveau DEA de physique.

Dans la fin de ce cours, $\mathrm{j}$ 'aborde la diffusion inélastique. C'est-à-dire le calcul de la fonction de structure lorsque l'énergie du neutron change lors de la collision. Ce cas sera traité dans la dernière session du cours.

\section{NOTATIONS UTILISÉES}

b longueur de diffusion du noyau

$b_{0}, \quad$ coefficients de la longueur de diffusion du noyau indépendant du spin

$b_{n} \quad$ coefficients de la longueur de diffusion du noyau dépendant du spin

$b^{+}, b^{+} \quad$ longueur de diffusion du noyau dépendant de l'état de spin du neutron

$b^{\prime \prime} \quad$ partie imaginaire de la longueur de diffusion du noyau

E l'énergie du neutron

e la charge de l'électron

$f(\mathbf{Q}) \quad$ facteur de forme de l'atome

$\mathbf{F}_{\mathrm{M}}(\mathbf{Q})$ ou $\mathbf{M}$ Facteur de structure magnétique (vecteur).

$F_{\mathrm{N}}(\mathbf{Q})$ ou $N \quad$ Facteur de structurenucléaire (scalaire).

$\mathrm{g} \quad$ le facteur de Landé, $(\mathrm{g}=2)$ 


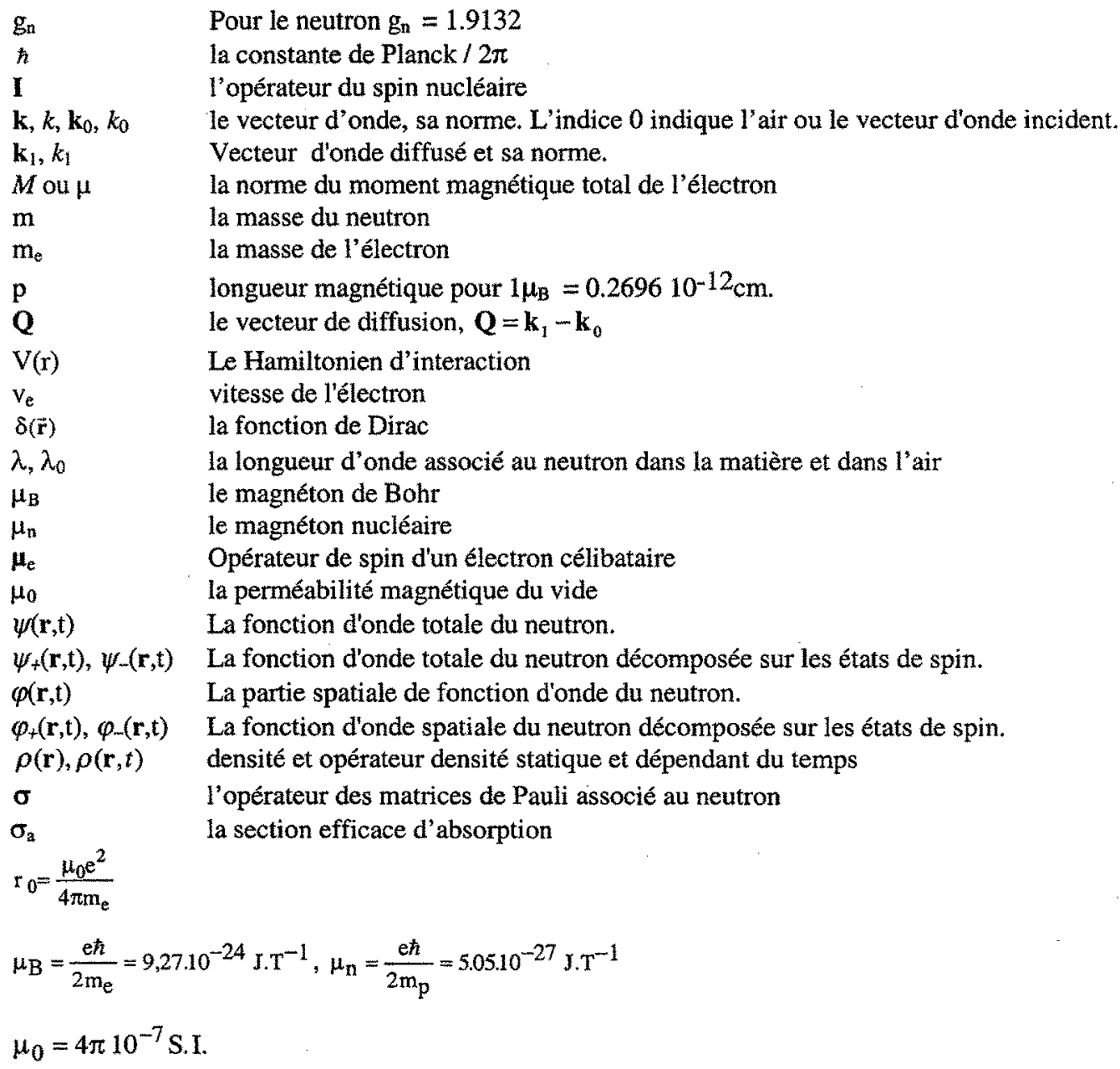

$\theta(x)$ est la fonction-saut de Heaviside définie par: $\left\{\begin{array}{lll}\theta(x)=1 & \text { quand } & x>0 \\ \theta(x)=1 / 2 & \text { quand } & x=0 \\ \theta(x)=0 & \text { quand } & x<0\end{array}\right.$

On appelle up (resp. down) la polarisation d'un neutron dont le spin est parallele (resp. antiparallèle) au champ magnétique appliqué.

Un processus spin-flip correspond à un neutron incident polarisé up (resp. down) qui devient polarisé down (resp. up) après diffusion.

Dans un processus non-spin-flip, le neutron a le même état de polarisation avant et aprèsdiffusion. 


\section{LE NEUTRON, ONDE}

\subsection{Equation de Schrödinger, paquet d'onde du neutron}

(L'essentiel de ce qui est dit ici se trouve dans tous les livres de mécanique quantique).

Une particule est décrite par une superposition linéaire d'ondes planes solutions de l'équation de Schrödinger :

$$
i \hbar \frac{\partial \psi}{\partial t}=\frac{-\hbar^{2}}{2 m} \Delta \psi+V(\mathbf{r}, t) \psi
$$

Dans le cas d'un potentiel $V$ ne dépendant pas du temps, on résout l'équation stationnaire en posant

$$
\psi(\mathbf{r}, t)=\varphi(\mathbf{r}) e^{-i \omega t}
$$

Ce qui donne, avec $E=\hbar \omega$

$$
\frac{-\hbar^{2}}{2 m} \Delta \varphi+V(\mathbf{r}) \varphi=E \varphi
$$

Dans le cas d'une particule libre $(V(\mathbf{r})=0)$,

la solution générale est de la forme (onde plane):

$$
\psi(\mathbf{r}, t)=A \cdot e^{i(\mathbf{k}, \mathbf{r}-\omega *)}
$$

la solution physique de (3) est donnée par une solution du type :

$$
\psi(\mathbf{r}, t)=\frac{1}{\sqrt{2 \pi}} \int g(\mathbf{k}) e^{i(\mathbf{k} \cdot \mathbf{r}-\omega(\mathbf{k}) t)} d \mathbf{k}
$$

où $\mathbf{g}(\mathbf{k})$ est centrée sur $\mathbf{k}_{0}$ de la particule et tend vers 0 ainsi que $\psi$ quand largument tend vers l'infini. (typiquement une Gaussienne)

L'onde plane monochromatique (4) n'est pas physique mais on fait en général les calculs en modélisant la particule par une onde plane en sachant que la vraie fonction est une combinaison linéaire d'ondes planes.

Le problème de la taille du paquet d'onde du neutron n'est pas simple à résoudre et actuellement, nous n'avons que des indications. On sépare la taille suivant un axe

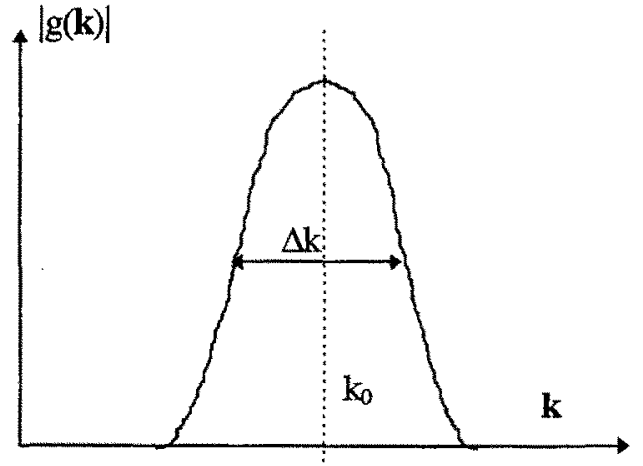


perpendiculaire au vecteur $\mathbf{k}_{0}$ (taille transversale du paquet d'onde) et la partie le long de $\mathbf{k}_{0}$.

A partir de la création du neutron dans le réacteur, l'absence d'interactions avec le vide fait qu'on s'attend à un paquet d'onde très grand au niveau de l'expérience et seule les collimations limitent celui-ci. Pratiquement, il semble que sa taille soit plutôt comprise entre 10 et $100 \mu \mathrm{m}$. Cela influencera d'ailleurs le traitement de la réflectivité mais pas du tout les expériences de petits angles ou de diffraction. Il faut aussi signaler que, par rapport à un rayonnement de lumière, un seul neutron interagit avec l'échantillon à un instant donné et donc notre question est la cohérence du neutron avec lui-même, deux neutrons ne sont pas cohérents entre eux.

\subsection{Introduction du spin}

Comme le neutron a un spin, la fonction d'onde associée se décompose sur la base des états du spin et s'écrit donc :

$$
|\psi\rangle=\psi_{+}(\mathbf{r}, t)|+\rangle+\psi_{-}(\mathbf{r}, t)|-\rangle
$$

les deux termes $\psi_{+}(\mathbf{r}, t)$ et $\psi_{-}(\mathbf{r}, t)$ sont de la forme donnée en (4).

La présence de ce spin explique la sensibilité du neutron au magnétisme.

\section{DIFFUSION SUR UN POTENTIEL}

\subsection{Potentiel fixe, indépendant du temps}

Ici on considère un potentiel indépendant du temps (une autre particule liée par exemple), fixé dans l'espace et qui décroît plus vite que $1 / \mathrm{r}^{3}$.

Le neutron diffuse de façon élastique
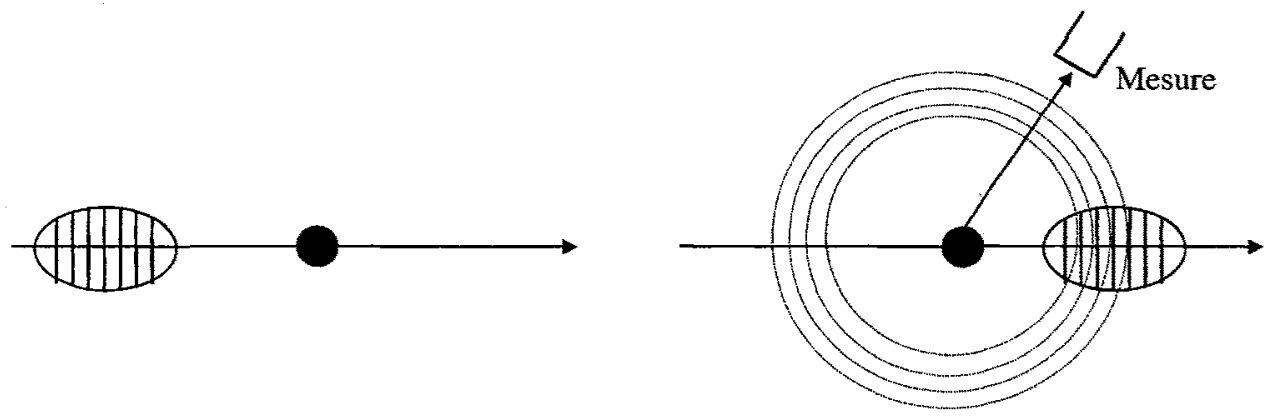

On recherche la solution de l'équation de Schrödinger pour ce potentiel, avec pour condition aux limites, un paquet d'onde correspondant au neutron libre loin de la zone d'action du potentiel.

On montre qu'à grande distance, l'état stationnaire de diffusion se comporte comme : 


$$
\varphi_{\text {dif }}(\mathbf{r}) \underset{r \rightarrow \infty}{\approx} e^{i \mathbf{k}_{0} r}+f(\Omega) \frac{e^{i k_{0} r}}{r}
$$

Le premier terme représente l'onde incidente, le deuxième terme l'onde diffusée.

La section efficace différentielle, qui est égale au nombre de particules dn comptées dans un détecteur d'angle solide $\mathrm{d} \Omega$ par unité de temps et de flux incident, est donnée simplement par

$$
\frac{d \sigma}{d \Omega}=|f(\Omega)|^{2}
$$

Cette section efficace différentielle est la quantité mesurée expérimentalement.

\subsection{Equation intégrale de la diffusion}

La formule (7) n'est pas sortie du chapeau mais découle directement du fait que

$$
\left(\Delta+k^{2}\right)\left(\frac{e^{ \pm i k r}}{r}\right)=-4 \pi \delta(r)
$$

(à titre informatif $\frac{-1}{4 \pi} \frac{e^{ \pm i k r}}{r}$ sont appelées fonction de Green de l'opérateur $\left(\Delta+k^{2}\right)$ )

Grâce à cette propriété, on montre que toute solution de l'équation de Schrödinger vérifie une équation intégrale de diffusion:

$$
\varphi_{d i j}(\mathbf{r})=e^{i \mathbf{k}_{0} \mathbf{r}}-\frac{m}{2 \pi \hbar^{2}} \int \frac{e^{i k\left|\mathbf{r}-\mathbf{r}^{\prime}\right|}}{|\mathbf{r}-\mathbf{r}|} V\left(\mathbf{r}^{\prime}\right) \varphi_{d i f}\left(\mathbf{r}^{\prime}\right) d^{3} \mathbf{r}^{\prime}
$$

et réciproquement

Pour des $\mathbf{r}$ grands, on développe au premier ordre $|\mathbf{r}-\mathbf{r}|$ et on obtient

$$
\varphi_{d i f}(\mathbf{r}) \approx e^{i k_{0} \pi}-\frac{m}{2 \pi h^{2}} \frac{e^{i k r}}{r} \int e^{-i k_{1} r^{\prime}} V\left(\mathbf{r}^{\prime}\right) \varphi_{d i f}\left(\mathbf{r}^{\prime}\right) d^{3} \mathbf{r}^{\prime}
$$

Ce qui donne le résultat avec

$$
f(\Omega)=-\frac{m}{2 \pi \hbar^{2}} \int e^{-i \mathbf{k}_{1} \mathbf{r}^{\prime}} V\left(\mathbf{r}^{\prime}\right) \varphi_{d i j}\left(\mathbf{r}^{\prime}\right) d^{3} \mathbf{r}^{\prime}
$$

Dans une notation mécanique quantique (11) s'écrit : 


$$
f(\Omega)=-\frac{m}{2 \pi \hbar^{2}}\left\langle\varphi_{\text {dif }}|V| \varphi_{\text {inc }}\right\rangle
$$

Dans la littérature, une onde plane définie par un vecteur $\mathbf{k}_{0}$ se note souvent $\left|\mathbf{k}_{0}\right\rangle$.

\subsection{Approximation de Born}

(11) est difficile à manier, Born a donc proposé un développement en réintroduisant (10) dans (11). En s'arrêtant au premier ordre, on arrive à :

$$
f(\Omega)=-\frac{m}{2 \pi h^{2}} \int e^{-i\left(\mathbf{k}_{1}-\mathbf{k}_{0}\right) r} V(\mathbf{r}) d^{3} \mathbf{r}
$$

$\mathbf{Q}=\mathbf{k}_{\mathbf{1}}-\mathbf{k}_{0}$ est appelé vecteur de diffusion

L'amplitude de diffusion est dans ce cas directement reliée à la transformée de Fourier du potentiel diffuseur.

\subsection{Validité de l'approximation de Born}

Cette approximation est d'autant plus valide que la fonction d'onde totale est peu différente de la fonction d'onde incidente : donc quand la diffusion par le potentiel est faible.

En diffraction, cette validité est remise en cause lorsque la diffusion sur un pic de Bragg est très intense. (diffusion multiple, extinction).

En réflectivité cette approximation est totalement fausse près de la réflectivité totale.

Pour la diffusion de neutrons aux petits angles, l'approximation est valable tant que l'intensité diffusée ou absorbée est faible par rapport au faisceau incident.

\section{L'INTERACTION NEUTRON-MATIÈRE}

Les deux principales interactions neutron-matière sont l'interaction forte avec le noyau et l'interaction magnétique avec les moments présents.

\subsection{L'interaction neutron-noyau}

\subsubsection{Pseudo-potentiel de Fermi}

La diffusion d'un neutron sur un noyau découle de l'interaction forte entre les deux particules :

Le potentiel $V$ est grand mais de portée beaucoup plus petite que la longueur d'onde du neutron. 
Cette propriété permet d'écrire ce potentiel sous la forme :

$$
V_{F}(\mathbf{r})=b\left(\frac{2 \pi \hbar^{2}}{m}\right) \delta(\mathbf{r})
$$

b est une grandeur mesurée expérimentalement, dépendant du noyau considéré, appelée longueur de diffusion.

Il dépend de plus du spin nucléaire I du noyau. Formellement, on l'écrit sous la forme d'un opérateur :

$$
b=b_{0}+\frac{1}{2} b_{n} 1 . \sigma
$$

On appelle souvent $b^{+}$et $b^{-}$les deux valeurs mesurées en fonction de l'état du spin du neutron $\mid+>$ ou $\mid \rightarrow$.

comme $\mathbf{J}=\mathbf{I}+1 / 2 \sigma$ est un bon nombre quantique, on obtient :

$$
\left\{\begin{array}{l}
b^{+}=b_{0}+\frac{1}{2} b_{n} I \\
b^{-}=b_{0}-\frac{1}{2} b_{n}(I+1)
\end{array}\right.
$$

\subsubsection{Absorption}

Il faut tenir compte de l'absorption possible du neutron, cela introduit, par le théorème optique, une partie imaginaire à $b, b^{\prime \prime}$ telle que

$$
b^{\prime \prime}=\left(k_{0} / 4 \pi\right)\left(\sigma_{a}\right)
$$

Le neutron est une sonde très transparente pour la matière et seuls quelques éléments ont une absorption non négligeable due à des résonances (le neutron a tendance à former un état lié avec le noyau). Ces noyaux sont le Cadmium, le bore, l'Hélium 3, le Gadolinium, le Samarium et le Lithium 6.

\subsection{L'interaction magnétique}

L'interaction magnétique est due à la réaction du moment magnétique du neutron au champ créé par les électrons non appariés de l'atome magnétique. Ce champ contient une partie orbitale et une partie de spin. La diffraction de neutrons ne permet pas de séparer les deux contributions.

$$
\mathbf{H}=\nabla \times\left\{\frac{\boldsymbol{\mu}_{e} \times \mathbf{r}}{r^{3}}\right\}-\frac{e \mathbf{v}_{e} \times \mathbf{r}}{c}
$$

L'interaction s'écrit : 


$$
V_{M}(\mathbf{r})=-g_{n} \mu_{n} \boldsymbol{\sigma} . \mathbf{H}
$$

$\mu_{e}=-2 \mu_{B} \mathrm{~s}$ est l'opérateur correspondant au spin de l'électron, $\mu_{\mathrm{B}}$ est le magnéton de Bohr, $\mathrm{v}_{\varepsilon}$ est la vitesse de l'électron.

\subsection{L'interaction Zeeman}

C'est l'interaction du neutron avec le champ magnétique extérieur, elle est de la même forme que (9).

$$
V_{z}(\mathbf{r})=-g_{n} \mu_{n} \boldsymbol{\sigma} \cdot \mathbf{H}_{0}
$$

\section{LE FACTEUR DE STRUCTURE ÉLASTIQUE}

\subsection{La fonction de structure, les corrélations de paires}

On se place ici dans l'approximation de Born. Cette partie est la base nécessaire pour la diffraction et la diffusion aux petits angles.

\subsubsection{Diffusion par un atome}

L'intensité diffusée est donnée par le carré de

$$
f(\Omega)=-\frac{m}{2 \pi \hbar^{2}} \int e^{-i Q \cdot r} V(\mathbf{r}) d^{3} \mathbf{r}
$$

Dans le cas d'un atome non magnétique, le potentiel s'écrit :

$$
V_{F}(\vec{r})=\frac{2 \pi \hbar^{2}}{m}\left(b_{0}+b_{n} \mathbf{I} . \boldsymbol{\sigma}\right) \delta(\vec{r})=\frac{2 \pi \hbar^{2}}{m} b \delta(\vec{r})
$$

d'où on obtient simplement $f(\Omega)=-b$

La partie magnétique de l'interaction donne :

$$
a_{M}\left(\mathbf{k}_{1}-\mathbf{k}_{0}\right)=-\frac{m}{2 \pi \hbar^{2}} \int e^{-i\left(\mathbf{k}_{1}-\mathbf{k}_{0}\right) \mathbf{r}} V_{M}(\mathbf{r}) d^{3} \mathbf{r}
$$

Le calcul détaillé de cette transformée de Fourier peut être trouvé dans de nombreux ouvrages On obtient :

$$
a_{M}(\mathbf{Q})=p \cdot(\mathbf{Q} \wedge \mathbf{M}(\mathbf{Q}) \wedge \mathbf{Q})=p \cdot \mathbf{M}_{\perp}(\mathbf{Q}) \cdot \boldsymbol{\sigma}
$$


$\mathbf{M}_{\perp}$ représente la composante perpendiculaire à $\mathbf{Q}$ de la transformée de Fourier de l'aimantation de l'atome considéré.

$$
\mathbf{M}(\mathbf{Q})=\int \mathbf{M}(\mathbf{r}) e^{i \mathbf{Q} \cdot r} d^{3} r
$$

$p$ est une constante numérique valant $2.696 .10^{-13} \mathrm{~cm} / \mu_{\mathrm{B}}$. Elle représente l'amplitude de diffusion pour un moment de $1 \mu_{\mathrm{B}}$ à $\mathbf{Q}=0$.

La formule (24) est tout à fait générale. Il faut imaginer que cela correspond à la brique élémentaire de la diffusion. La diffusion sur une assemblée d'atomes ne sera qu'une somme pondérée de ce type de termes.

Lorsque $\mathbf{Q}$ tend vers $\mathbf{0}, \mathbf{M}(\mathbf{Q})$ tend vers le moment total de l'atome (voir (25)) mais lorsque $\mathbf{Q}$ s'éloigne de 0 , l'interaction va dépendre de la forme du moment magnétique. Cette forme est intimement liée à l'atome et à son environnement. Le calcul complet des facteurs de forme est très lourd [1]. La chose à retenir est la décroissance du facteur de forme pour $\mathbf{Q}$ grand. Un exemple de facteur de forme sera donné dans la partie traitant de la polarimétrie.

Pour tenir compte de ce facteur de forme dans les équations, on écrit souvent :

$$
\mathbf{M}_{\perp}(\mathbf{Q})=\mathbf{m}_{\perp} f(\mathbf{Q})
$$

En conclusion, l'amplitude de diffusion du neutron par un atome s'écrit :

$$
a(\mathbf{Q})=b+p f(\mathbf{Q}) \mathbf{m}_{\perp}, \boldsymbol{\sigma}
$$

avec $p=2.696 .10^{-13} \mathrm{~cm} / \mu_{\mathrm{B}}$.

\subsubsection{Diffusion par une assemblée d'atomes}

Pour un ensemble d'atomes, on peut calculer l'onde diffusée soit à partir de la formulation première de la diffusion (eq 7,8) soit directement à partir de l'approximation de Born. En général, c'est la deuxième qui est donnée dans les manuels. La première permet de sentir les approximations faites notamment le fait que les source/ détecteur sont considérés comme très loin par rapport à la taille du système diffusant.

\section{Calcul à partir de la fonction d'onde}

L'onde diffractée par une assemblée de noyaux sera la somme des ondes diffractée par chacun corrigée par un facteur de phase de l'onde incidente. On obtient alors dans l'approximation de Born :

$$
\varphi(\mathbf{r})=\exp \left(i \mathbf{k}_{0} . \mathbf{r}\right)-\sum_{i} a_{i} \frac{\exp \left(i \mathbf{k}_{1} \mid \mathbf{r}-\mathbf{r}_{i}\right)}{\left|\mathbf{r}-\mathbf{r}_{i}\right|} \cdot \exp \left(-i \mathbf{k}_{0} . \mathbf{r}_{i}\right) .
$$

On développe au premier ordre dans l'exponentielle et à l'ordre 0 pour $1 / r$ car le premier sera beaucoup sensible à des différences de l'ordre de $1 / k$ c'est-à-dire quelques $\AA$, pour des neutrons thermiques.

\footnotetext{
${ }^{1}$ W. Marshall and S.W. Lovesey, Theory of thermal neutron scattering, Clarendon Press Oxford 1975
} 
On a au premier ordre $\left|\mathbf{r}-\mathbf{r}_{i}\right|=r-\left(\mathbf{r}_{i} \cdot \mathbf{r}\right) / r$.

On trouve alors que

$$
\left.\varphi(\vec{r})=\exp \left(i \mathbf{k}_{0} \cdot \mathbf{r}\right)-\frac{\exp \left(i k_{d} r\right)}{r} \sum_{i} a_{i} \cdot \exp \left(-i \mathbf{Q} \cdot \mathbf{r}_{i}\right)\right)
$$

\section{Calcul direct}

Le potentiel total est donné par

$$
V(\mathbf{r})=\sum_{i} b_{i} \delta\left(\mathbf{r}-\mathbf{r}_{i}\right)-g_{n} \mu_{n} \sigma . H_{i}\left(\mathbf{r}-\mathbf{r}_{i}\right)
$$

En prenant la transformée de Fourier (20) on arrive à

$$
f(\Omega)=-\sum_{i} \int e^{-i Q \cdot r} b_{i} \delta\left(\mathbf{r}-\mathbf{r}_{i}\right) d^{3} \mathbf{r}+\int e^{-i Q \cdot r} g_{n} \mu_{n} \boldsymbol{\sigma} . \mathbf{H}_{i}\left(\mathbf{r}-\mathbf{r}_{i}\right) d^{3} \mathbf{r}
$$

d'où

$$
f(\Omega)=-\sum_{i} e^{-i \mathbf{Q} \cdot x_{i}}\left(b_{i}+p \sigma \mathbf{M}_{i 1}(Q)\right)=-\sum_{i} a_{i} e^{-i Q \cdot r_{i}}
$$

La section efficace différentielle élastique est égale à :

$$
\Sigma=\frac{d \sigma}{d \Omega}=|f(\Omega)|^{2}=\sum_{i, j} a_{i} a_{j}^{*} \cdot \exp \left(-\mathbf{Q}\left(\mathbf{r}_{i}-\mathbf{r}_{j}\right)\right)
$$

avec

$$
\mathbf{Q}=\mathbf{k}_{1}-\mathbf{k}_{0} \text { et } Q=\frac{4 \pi}{\lambda} \sin (\theta / 2)
$$

où $\theta$ est l'angle entre le faisceau incident et le faisceau diffusé (direction d'observation)

de plus $a_{i}(\mathbf{Q})=b_{i}+p f_{i}(\mathbf{Q}) \mathbf{m}_{i \perp} . \sigma$

Exemple pour une diffusion aux petits angles.

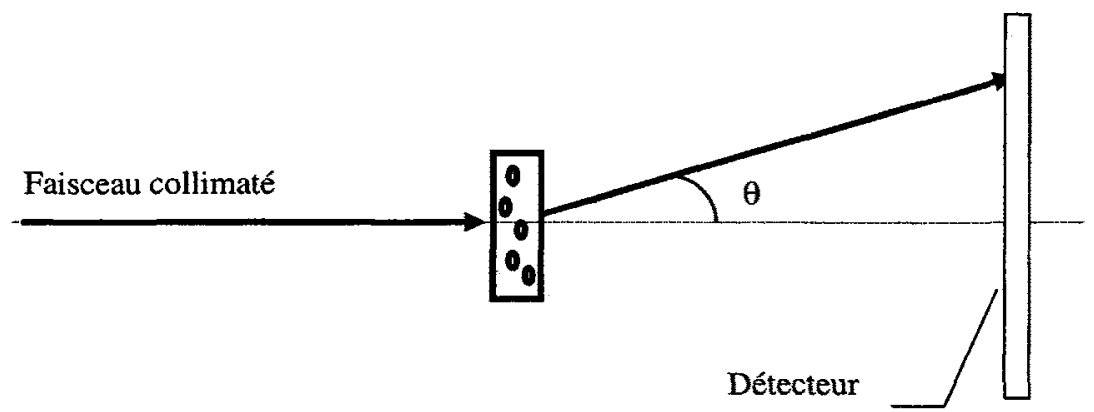




\subsection{Facteur de structure nucléaire, facteur de structure magnétique}

La formule (33) peut être réécrite légèrement différemment.

$$
\frac{d \sigma}{d \Omega}=\left|F_{N}(\mathbf{Q})+\mathbf{F}_{M}(\mathbf{Q}) \cdot \sigma\right|^{2}
$$

avec

$$
F_{N}(\mathbf{Q})=\sum_{i} b_{i} e^{i \mathbf{Q} \cdot r_{i}} \text { et } \mathbf{F}_{M}(\mathbf{Q})=\sum_{i} p \mathbf{m}_{i \perp} f_{i}(\mathbf{Q}) e^{i \mathbf{Q} \cdot r_{i}}
$$

Ces deux termes sont appelés facteurs de structure nucléaire et facteurs de structure magnétique. Il faut faire attention que le facteur de structure magnétique est un vecteur.

De plus, ces facteurs peuvent être complexes. Seule la mesure qui est le module au carré est une observable et donc réelle.

\subsubsection{Cas de neutrons polarisés avec analyse}

Dans le cas de neutrons polarisés, (on verra dans le cours de réflectivité comment on peut obtenir des faisceau très bien polarisés) les neutrons sont presque tous dans l'état $|+\rangle$ (up) c'est-à-dire alignés avec le champ magnétique ou dans l'état $|-\rangle$ (down) c'est-à-dire alignés dans un sens opposé au champ.

Remarque : en cas de passage soudain en champ nul, le neutron reste dans l'état juste avant le passage. C'est cet état qui sert de référence (axe de quantification).

On peut dans l'absolu mesurer la diffusion pour n'importe quelle direction du spin incident et pour n'importe quelle direction du spin diffusé.

Ici je vais juste traiter le cas où un champ magnétique est appliqué dans l'environnement de l'échantillon.

Dans ce cas, ce champ fixe l'axe de polarisation et donc de quantification du spin du neutron.

On peut à ce moment, mesurer uniquement 4 grandeurs : le neutron arrive up ou down et peut être uniquement diffusé up ou down.

A partir des matrices de Pauli données en annexe 2 on peut rapidement montrer que

$$
\begin{aligned}
& \frac{d \sigma^{++}}{d \Omega}=\left|F_{N}(\mathbf{Q})+F_{M z}(\mathbf{Q})\right|^{2}, \frac{d \sigma^{-}}{d \Omega}=\left|F_{N}(\mathbf{Q})-F_{M z}(\mathbf{Q})\right|^{2} \\
& \frac{d \sigma^{+-}}{d \Omega}=\frac{d \sigma^{+}}{d \Omega}=\left|F_{M x}(\mathbf{Q})\right|^{2}+\left|F_{M y}(\mathbf{Q})\right|^{2}
\end{aligned}
$$

Il ne faut pas oublier que le facteur de structure magnétique n'est sensible qu'à la composante de l'aimantation perpendiculaire à $\mathbf{Q}$. 


\subsubsection{Cas de neutrons polarisés sans analyse}

Si le spectromètre ne permet pas d'analyser les neutrons, pour des neutrons envoyés up ou down, on récolte la somme des neutrons après diffusion. On mesure donc 2 quantités :

$$
\begin{aligned}
& \frac{d \sigma^{+}}{d \Omega}=\left|F_{N}(\mathbf{Q})+F_{M z}(\mathbf{Q})\right|^{2}+\left|F_{M x}(\mathbf{Q})\right|^{2}+\left|F_{M y}(\mathbf{Q})\right|^{2}, \\
& \frac{d \sigma^{-}}{d \Omega}=\left|F_{N}(\mathbf{Q})-F_{M z}(\mathbf{Q})\right|^{2}+\left|F_{M x}(\mathbf{Q})\right|^{2}+\left|F_{M y}(\mathbf{Q})\right|^{2}
\end{aligned}
$$

\subsubsection{Cas de neutrons non polarisés}

Dans ce cas, on ne mesure bien sur qu'une quantité qui est la somme des quantités ci-dessus :

$$
\frac{d \sigma}{d \Omega}=\left|F_{N}(\mathbf{Q})\right|^{2}+\left|F_{M x}(\mathbf{Q})\right|^{2}+\left|F_{M y}(\mathbf{Q})\right|^{2}+\left|F_{M z}(\mathbf{Q})\right|^{2}=\left|F_{N}(\mathbf{Q})\right|^{2}+\left|\mathbf{F}_{M}(\mathbf{Q})\right|^{2}
$$

\section{LE CAS GÉNÉRAL, DIFFUSION INÉLASTIQUE}

Dans le cas le plus général, le système peut changer d'état lors de la diffusion. Il peut alors donner ou recevoir de l'énergie du neutron. Le neutron va donc être vu comme interagissant avec une quasiparticule décrivant une excitation du système (phonon, onde de spin) ou bien comme un rayonnement capable d'induire une transition entre deux niveaux (niveaux de vibration ou de rotation par exemple). Ci-dessous est donné un exemple de courbe de dispersion d'une onde de spin dans du fer. Un intérêt des neutrons pour ces études est d'être sélectif en vecteur d'onde : on sonde une excitation ayant un vecteur $\mathbf{Q}$ de propagation. La résonance ferromagnétique par exemple n'est capable de sonder que le vecteur $\mathbf{Q}=\mathbf{0}$.

Pour calculer l'amplitude totale de diffusion, il faut sommer toutes les amplitudes de diffusion pondérées par les probabilités d'existence de l'état initial et final. La seule contrainte est la conservation de l'énergie lors de cette diffusion.

On va écrire une double section différentielle sous la forme d'une somme :

$$
\left(\frac{d^{2} \sigma}{d \Omega d E}\right)_{\mathbf{k}_{0} \sigma_{0} \rightarrow \mathbf{k}_{1} \sigma_{1}}=A \sum_{\lambda_{0}, \lambda_{1}} p_{\lambda_{0}}\left|\left\langle\mathbf{k}_{1} \sigma_{1} \lambda_{1}|V| \mathbf{k}_{0} \sigma_{0} \lambda_{0}\right\rangle\right|^{2} \delta\left(E+E_{\lambda_{0}}-E_{\lambda_{1}}\right)
$$

$\lambda_{0}$ représente l'état initial du système qui a une certaine probabilité d'exister $p_{\lambda_{0}}$ et dont l'énergie est $E_{\lambda_{0}}$. De même $\lambda_{1}$ représente l'état final du système.

$\mathbf{k}_{0} \sigma_{0}$ représente le neutron incident (vecteur d'onde et spin), $\mathbf{k}_{l} \boldsymbol{\sigma}_{l}$ représente le neutron diffusé.

$$
A=\left(\frac{1}{N}\right) \frac{k_{1}}{k_{0}}\left(\frac{m}{2 \pi \hbar^{2}}\right)
$$

Cette formule (40) n'est pas utilisable telle quelle et elle sera développée en fonction du problème particulier qui se posera. 
Le développement dans le cas des ondes de spin est bien fait dans la réf 2 p 80-82

Très souvent, on sera amené à décrire l'équation 40 en termes de fonctions de corrélations. C'est le cas des liquides ou de solides dans lesquels le concept de sites en équilibre n'est plus valide.

On définit à ce moment une fonction de corrélation dépendant du temps ou fonction de corrélation dynamique par

$$
G(\mathbf{r}, t)=\frac{1}{N} \int\left\langle\rho\left(\mathbf{r}^{\prime}-\mathbf{r}, 0\right) \rho\left(\mathbf{r}^{\prime}, t\right)\right\rangle d \mathbf{r}^{\prime}
$$

L'opérateur densité est défini par:

$$
\rho(\mathbf{r})=\sum_{i} \delta\left(\mathbf{r}-\mathbf{r}_{i}(t)\right)
$$

Exactement de la même façon que pour les fonction de corrélation statiques, on peut montrer que l'intensité mesurée est proportionnelle à la double transformée de Fourier de cette fonction de corrélation :

$$
S(\mathbf{Q}, \omega)=\iint e^{i \mathbf{Q} \cdot r-i \omega t} G(\mathbf{r}, t) d \mathbf{r} d t
$$

Cette fonction est appelée, fonction de structure dynamique.

\section{QUELQUES LIVRES UTILES}

\subsection{Mécanique quantique}

C. Cohen-Tannoudji, B. Diu, F. Laloë, Mécanique quantique, Collection enseignement des sciences, Hermann

\subsection{Diffusion, diffraction de neutrons}

G.E. Bacon, Neutron diffraction, Clarendon Press Oxford 1975.

W. Marshall and S.W. Lovesey, Theory of thermal neutron scattering, Clarendon Press Oxford 1975

Série Methods of experimental physics, vol 23- part $\mathrm{A}, \mathrm{B}$ et $\mathrm{C}$ (très complet).

Squires, "Introduction to the theory of thermal neutron scattering" Cambridge university Press.

\footnotetext{
${ }^{2}$ D. Price and K. Sköld, "Introduction to neutron scattering" dans methods of experimental physics, vol 23- part A, p $80-82$
} 


\section{ANNEXES}

\subsection{Annexe 1: Densité et fonction de corrélation de paires statique}

On considère un système, pouvant présenter des fluctuations de densité. Un liquide ou un ensemble de polymères en solutions sont de bons exemples.

Je définis ici les densités et fonctions de corrélation de paires à partir de distributions.

\subsubsection{Densité}

La densité est définie par : $\rho(\mathbf{r})=\sum_{i} \delta\left(\mathbf{r}-\mathbf{r}_{i}\right)\left(\mathrm{cm}^{-3}\right)$

A partir de cette formule on peut retrouver l'intensité donnée dans l'encadré page 10.

\subsubsection{Fonction de corrélation de paires}

Elle est définie par

$$
g(\mathbf{r})=\frac{V}{N^{2}} \sum_{i, j \neq i}<\delta\left(\mathbf{r}-\mathbf{r}_{i}+\mathbf{r}_{j}\right)>
$$

ou bien par

$$
g(\mathbf{r})=\frac{V^{2}}{N^{2}} \sum_{i, j \neq i}<\delta\left(\mathbf{r}-\vec{r}_{i}\right) \delta\left(-\mathbf{r}_{j}\right)>=\frac{n^{(2)}(\mathbf{r}, 0)}{n^{2}}
$$

Ces deux fonctions sont égales dans le cas d'une invariance par translation du milieu les $\langle>$ signifient une moyenne statistique sur les configurations possibles. Cette moyenne est en général spatiale mais elle peut être aussi temporelle.

Si on remarque que :

$$
\int \exp (-i \mathbf{q} \cdot \mathbf{r}) .<\delta\left(\mathbf{r}-\mathbf{r}_{i}+\mathbf{r}_{j}\right)>d^{3} \mathbf{r}=\left\langle\exp \left(-i \mathbf{q} \cdot\left(\mathbf{r}_{i}-\mathbf{r}_{j}\right)\right)>\right.\text {. }
$$

on a

$$
\Sigma=\int \exp (-i \mathbf{q} \cdot \mathbf{r}) \cdot \sum_{i, j} b_{i} b_{j}<\delta\left(\mathbf{r}-\mathbf{r}_{i}+\mathbf{r}_{j}\right)>d^{3} \mathbf{r}
$$

et donc si les $b_{i}$ sont égaux :

$$
\frac{\Sigma}{V}=\frac{N}{V} b^{2}+b^{2} \frac{N^{2}}{V^{2}}\left(\int d^{3} \vec{r} \exp (-i \vec{q} \cdot \vec{r})(g(\vec{r})-1)+(2 \pi)^{3} \delta(\vec{q})\right)
$$




\subsection{Annexe 2 : matrices de Pauli}

Matrices de Pauli.

Les matrices de Pauli sont l'expression de l'opérateur de Pauli $\sigma$. elles ont pour valeur :

$$
\left\{\begin{array}{l}
\sigma_{x}=\left(\begin{array}{cc}
0 & 1 \\
1 & 0
\end{array}\right) \\
\sigma_{y}=\left(\begin{array}{cc}
0 & -i \\
i & 0
\end{array}\right) \\
\sigma_{z}=\left(\begin{array}{cc}
1 & 0 \\
0 & -1
\end{array}\right)
\end{array}\right.
$$

Ces matrices sont exprimées dans la base naturelle du spin $1 / 2|+\rangle=\left(\begin{array}{l}1 \\ 0\end{array}\right) \mathrm{et}|-\rangle=\left(\begin{array}{l}0 \\ 1\end{array}\right)$.

Cette base naturelle est celle où le spin est quantifié le long de $z$.

Dans le cas du neutron, $\mathrm{z}$ est la direction d'application du champ magnétique.

On peut ainsi calculer les relations :

$$
\sigma_{x}|+\rangle=|-\rangle, \sigma_{x}|-\rangle=|+\rangle, \sigma_{y}|+\rangle=-i|-\rangle, \sigma_{y}|-\rangle=i|+\rangle, \sigma_{z}|+\rangle=|+\rangle, \sigma_{z}|-\rangle=-|-\rangle
$$

